\title{
Immunoscore Combining CD8, FoxP3 and CD68 Expression and Distribution Predicts the Prognosis of Head and Neck Cancer Patients
}

\section{Sonia Furgiuele}

University of Mons Faculty of Medicine and Pharmacy: Universite de Mons Faculte de Medecine et Pharmacie

\section{Géraldine Descamps}

University of Mons Faculty of Medicine and Pharmacy: Universite de Mons Faculte de Medecine et Pharmacie

\section{Jérôme R. Lechien}

Hospital Foch: Hopital Foch

\section{Didier Dequanter}

CHU Saint-Pierre: Centre Hospitalier Universitaire Saint-Pierre

\section{Fabrice Journe}

University of Mons Faculty of Medicine and Pharmacy: Universite de Mons Faculte de Medecine et Pharmacie

Sven Saussez ( $\nabla$ sven.saussez@umons.ac.be)

University of Mons https://orcid.org/0000-0002-3655-1854

Original Article - Clinical Oncology

Keywords: Immunoscore, CD8, FoxP3, CD68, head and neck cancer, prognosis

Posted Date: February 4th, 2021

DOl: https://doi.org/10.21203/rs.3.rs-176581/v1

License: (c) (i) This work is licensed under a Creative Commons Attribution 4.0 International License. Read Full License 


\section{Abstract}

Purpose: The objective of this study was to assess immune cell infiltrates to develop an immunoscore for prognosis and to investigate its correlation with clinical data of patients with head and neck squamous cell carcinoma

Methods: CD8, FoxP3 and CD68 were evaluated by immunohistochemistry in 258 carcinoma samples and counted in stromal and intra-tumoral compartments. Optimal cut-offs were assessed to divide population regarding to survival while the prognostic value was established by using Kaplan-Meier curves and Cox regression models for each immune marker alone and in combination.

Results: We found with univariate analysis that infiltration of immune cells in both compartments was predictive for RFS and OS. Multivariate analysis revealed that CD8+ number influenced independently patient prognosis. Additionally, the combination of CD8, FoxP3 and CD68 in an immunoscore provided a significant association with OS ( $p=0.002, H R=9.87)$. Such immunoscore stayed significant $(p=0.018$, $\mathrm{HR}=11.17)$ in a multivariate analysis in comparison to tumour stage and histological grade which had lower prognostic values.

Conclusion: Altogether, our analysis indicated that an immunoscore including CD8, FoxP3 and CD68 immunostaining was a strong, independent, and significant prognostic marker which could be introduced into the landscape of current tools to improve the clinical management of head and neck cancer patients.

\section{Introduction}

Head and neck squamous cell carcinomas (HNSCC) are among the most prevalent cancers worldwide, setting them in the 6th place (Ferlay et al. 2019). In Belgium, their incidences are higher and such cancers arise at the 4th position in men (Jerome R. Lechien et al. 2019). Despite advances in therapeutic approaches, the mortality rate has remained relatively constant in recent years, with a 5-year survival rate around $50 \%$ and recurrences occurring in $40-60 \%$ of treated patients (Windon et al. 2018). This poor response to treatment can be explained in part by late diagnosis and a lack of efficient drugs in the case of tumour recurrence. However, it appears that the cell composition of the tumour microenvironment (TME) is likely to influence patient outcome (Valkenburg, de Groot, and Pienta 2018). The well-known risk factors of HNSCC are the consumption of alcohol and tobacco, as well as infection with the human papillomavirus (HPV), which is known to be associated with a better prognosis for the youngest patients with oropharyngeal carcinoma (Young et al. 2015). In this context, several studies suggest that HPV + patients with HNSCC have a specific TME which may influence the response to treatment (Mirza et al. 2019; Kindt et al. 2016; Seminerio et al. 2018; Seminerio et al. 2019).

Macrophages and more specifically tumour-associated macrophages (TAMs) are the most abundant cells within the TME and they are able to stimulate regulatory T lymphocytes (Treg) cells to switch to a pro-tumour environment (Evrard et al. 2019; Jérôme R. Lechien et al. 2020). Regarding macrophages, we previously showed that CD68 + infiltration arises during HNSCC progression in the intra-tumoral (IT) 
compartment and is associated with the tumour stage. We also highlighted that CD $68+$ recruitment is higher in HPV + patients than in HPV- ones. Moreover, a high infiltration of CD68 + cells was related to a shorter recurrence-free survival (RFS) as well as a shorter overall survival (OS) (Seminerio et al. 2018).

Immune surveillance is also governed by the tumour-infiltrating T lymphocytes (TILs) (de Ruiter et al. 2017). Among them, the CD $8+T$ lymphocytes act specifically on the cancer cells in order to eliminate them (S. M. Y. Chen et al. 2020). In HNSCC, a high density of CD8 + is correlated with a good prognosis (Lechner et al. 2017). Concerning Treg, which are characterized by the transcription factor forkhead box P3 (FoxP3) (Sakaguchi et al. 2010), we previously showed that FoxP3 + Treg infiltration increased during HNSCC progression (from dysplasia to carcinoma) and that tumours with high Treg infiltration were associated with longer RFS and OS (Seminerio et al. 2019; Kindt et al. 2017).

In advanced HNSCC, the gold standard treatment remains the concomitant chemoradiotherapy, but the emergence of immunotherapy over the last years has changed the landscape of HNSCC treatment (Lyford-Pike et al. 2013; Gavrielatou et al. 2020). However, using anti-cancer drug like immunotherapy is challenging due to the heterogeneity of the TME composition (Canning et al. 2019). Importantly, HPV status is now included in the tumour-node-metastasis (TNM) staging system (Leemans, Snijders, and Brakenhoff 2018) indicating the importance of additional prognostic information to propose the most appropriate treatment for patients. Currently, there is no immune-based classification of head and neck cancer. However, the evaluation of immune cell recruitment to classify HNSCC patients in different immunologic subgroups depending on the TME composition could be helpful to improve patient prognosis. The value of immunoscore has already been highlighted in several cancers, such as cervical cancer (H. Chen et al. 2020), hepatocellular carcinoma (Petrizzo and Buonaguro 2016), melanoma (Nie et al. 2019), colon cancer (Pagès et al. 2018), gastric cancer (Jiang et al. 2018), pancreatic cancer (Tahkola et al. 2018) and lung cancer (Gao et al. 2020), and it is starting to draw attention in HNSCC. On this point, Zhang et al. recently showed the interest of $C D 3+$ and $C D 8+$ cell infiltration scoring in combination with the TNM staging system in HNSCC patients (Zhang et al. 2018), and we reported that high stromal FoxP3 $+\mathrm{T}$ cell number combined with tumour stage improved prognosis in HNSCC patients (Kindt et al. 2017).

In this study, we propose an immune signature based on CD8+, FoxP3 + and CD68 + count in IT and/or stromal (ST) compartments in a large clinical series of 258 patients with HNSCC. The immunoscore is compared to tumour stage and histological grade using multivariate analyses.

\section{Methods}

\section{Patients and clinical data}

A total of 258 patients presenting HNSCC were enrolled on our study. The Table 1 describes the clinicopathological characteristics, treatment, and follow-up data. Formalin-fixed paraffin-embedded specimens obtained after surgical resection at Saint-Pierre Hospital (Brussels, Belgium), Jules Bordet 
Institute (Brussels, Belgium), EpiCURA Baudour Hospital (Baudour, Belgium) and CHU Sart-Tilman (Liège, Belgium) between 2002 and 2019 were used for immunohistochemical labelling. This retrospective study has been approved by the Institutional Review Board (Jules Bordet Institute, number CE2319). 
Table 1

Patient population characteristics

\section{Variables}

Age (years)

Median (range)

Recurrence-free survival (RFS)

(months)

Median (range)

Yes

No

Unknown

Overall survival (OS) (months)

Median (range)

Alive

Dead

Unknown

Gender

Male

Female

Anatomic site

Oral cavity

Oropharynx

Larynx

Hypopharynx

Nasopharynx

Tumour stage
Number of cases

$n=258$

$61(29-90)$

$p$ value
Relapse-free survival Overall survival

$\mathrm{HR}(95 \% \mathrm{Cl})$ $p-$
value
$22(1-245)$

104

120

34

$33(1-294)$

124

102

32

\begin{tabular}{|c|c|c|}
\hline $0.048^{*}$ & $\begin{array}{l}0.67(0.45- \\
0.99)\end{array}$ & 0.943 \\
\hline
\end{tabular}

177

81

113

80

44

19

2
0.274
$1.27(0.99-$ 2.82)
$1.91(1.22-$
$3.00)$

${ }^{*}$ Bold $p$-values indicated significant correlations (Cox regression) 


\begin{tabular}{|c|c|c|c|c|c|}
\hline Variables & $\begin{array}{l}\text { Number of } \\
\text { cases }\end{array}$ & \multicolumn{2}{|c|}{ Relapse-free survival } & \multicolumn{2}{|c|}{ Overall survival } \\
\hline$I-I I$ & 84 & & & & \\
\hline III-IV & 130 & & & & \\
\hline Unknown & 44 & & & & \\
\hline Histological grade & & 0.225 & $\begin{array}{l}0.76(0.45- \\
0.99)\end{array}$ & $0.029 *$ & $\begin{array}{l}0.62(0.40- \\
0.95)\end{array}$ \\
\hline Poorly differentiated & 112 & & & & \\
\hline Well differentiated & 89 & & & & \\
\hline Unknown & 57 & & & & \\
\hline Tumour invasion & & 0.053 & $\begin{array}{l}1.68(0.99- \\
2.82)\end{array}$ & 0.157 & $\begin{array}{l}1.42(0.88- \\
2.30)\end{array}$ \\
\hline Yes & 154 & & & & \\
\hline No & 59 & & & & \\
\hline Unknown & 45 & & & & \\
\hline \multicolumn{6}{|l|}{ Risk factors } \\
\hline Tobacco & & 0.326 & $\begin{array}{l}1.32(0.76- \\
2.29)\end{array}$ & 0.366 & $\begin{array}{l}1.29(0.74- \\
2.24)\end{array}$ \\
\hline Smoker & 181 & & & & \\
\hline Non-Smoker & 36 & & & & \\
\hline Unknown & 41 & & & & \\
\hline Alcohol & & 0.811 & $\begin{array}{l}1.05(0.69- \\
1.60)\end{array}$ & 0.445 & $\begin{array}{l}1.18(0.77- \\
1.81)\end{array}$ \\
\hline Drinker & 129 & & & & \\
\hline Non-Drinker & 78 & & & & \\
\hline Unknown & 51 & & & & \\
\hline HPV status & & 0.131 & $\begin{array}{l}0.65(0.37- \\
1.14)\end{array}$ & 0.562 & $\begin{array}{l}0.86(0.51- \\
1.44)\end{array}$ \\
\hline Positive & 65 & & & & \\
\hline Negative & 138 & & & & \\
\hline Unknown & 55 & & & & \\
\hline
\end{tabular}




\begin{tabular}{|c|c|c|c|c|c|}
\hline \multirow{2}{*}{$\begin{array}{l}\text { Variables } \\
\text { p16 status }\end{array}$} & \multirow{2}{*}{$\begin{array}{l}\text { Number of } \\
\text { cases }\end{array}$} & \multicolumn{2}{|c|}{ Relapse-free survival } & \multicolumn{2}{|c|}{ Overall survival } \\
\hline & & 0.103 & $\begin{array}{l}0.57(0.29- \\
1.12)\end{array}$ & 0.152 & $\begin{array}{l}0.62(0.32- \\
1.19)\end{array}$ \\
\hline Positive & 37 & & & & \\
\hline Negative & 121 & & & & \\
\hline Unknown & 100 & & & & \\
\hline
\end{tabular}

\section{HPV status}

DNA extractions were performed on formalin-fixed paraffin-embedded tissue (10 x $5 \mu \mathrm{m})$ with the QIAmp DNA Mini Kit (Qiagen, Benelux, Belgium), according to the manufacturer's recommended protocol. The HPV status of each patient was established by qPCR at the Algemeen Medisch Laboratorium (Antwerp, Belgium). Moreover, to determine the transcriptional activity of HPV, p16 immunostaining was performed using the recommended mouse monoclonal antibody (CINtec p16, clone E6H4, Ventana, Tucson, AZ, USA) on an automated immunostainer (BenchMark, Ventana, Roche) at the Jules Bordet Institute (Brussels, Belgium) as previously described (Kindt et al. 2016). The expression of p16 was defined as positive only when both the nucleus and the cytoplasm were stained and over $70 \%$ of the tumour cells were stained.

\section{Immunohistochemistry}

The $5 \mu \mathrm{m}$ thick slices of HNSCC were deparaffinized in toluene and rehydrated in a graded series of alcohols, then peroxidase was blocked using $\mathrm{H}_{2} \mathrm{O}_{2}$ and finally slices were rinsed with water for $7 \mathrm{~min}$. Antigen retrieval was processed by immersing the samples in $10 \% \mathrm{EDTA} / \mathrm{H}_{2} \mathrm{O}$ or in $10 \%$ citrate $/ \mathrm{H}_{2} \mathrm{O}$ followed by heating in a pressure cooker or in a microwave (buffer and timing are dependent on antibodies, see Supplementary Table 1). Non-specific sites were blocked with $0.5 \%$ caseine for $15 \mathrm{~min}$. Slices were incubated with primary antibody (anti-human CD68 monoclonal mouse, dilution 1:200, and anti-human CD8 monoclonal mouse, dilution 1:200, both from Dako) for 1 hour at room temperature or overnight at $4^{\circ} \mathrm{C}$. Kit PowerVision Poly-HRP IgG were used for the second antibody. For FoxP3 immunostaining, the anti-human FoxP3 monoclonal mouse (dilution 1:200, from Invitrogen) was used and the detection of this primary antibody was performed with the CSAll kit (Dako). For each immunohistochemistry, tonsil tissue was used as positive (and negative (no primary antibody)) controls.

\section{Calculation of an immunoscore}

The number of each immune cell type was counted in 5 fields in the IT and ST compartments with an Axio-Cam MRC5 optical microscope (Zeiss, Hallbergmoos, Germany) at 400x magnification by two 
investigators (S.F. and G.D.). The mean of each counting was calculated for each patient. For each marker in IT and ST, the cut-off value giving the best separation between two groups (HR and p for OS) was evaluated using the RStudio software. Then, if the mean number of the 5 fields was greater than the cut-off, the case was considered as "high" and if it was lower, the case was considered as "low". Based on such cut-offs, the prognostic value of each immune marker was examined regarding RFS and OS. From these analyses, an immunoscore was defined combining the most significant immune markers.

\section{Statistical analyses}

The optimal cut-off points of the population for each immuno-biomarker were calculated by using RStudio software (package from Cutoff Finder web application). Collected data were analysed with IBM SPSS software (version 23) (IBM, Ehningen, Germany). RFS and OS analyses were performed using Kaplan-Meier curves. Univariate and multivariate Cox regression models were applied to calculate the hazard ratio (HR), 95\% confidence interval and significance. $p$-values $<0.05$ were considered as significant.

\section{Results}

\section{Correlations between clinical characteristics and RFS or OS}

Our clinical series included a total of 258 HNSCC patients, among which 177 (68.6\%) were men and 81 (31.4\%) were women, with a median age of 61 years old (range, 29-90). Among these patients, 104 patients presented tumour recurrence and 102 died. The clinicopathologic characteristics are provided in the Table 1.

We evaluated the association between tumour stage, histological grade, tumour invasion or risk factors with RFS or OS. Cox regression models highlighted that among such parameters only tumour stage and histological grade correlated with OS. Evaluating Kaplan-Meier survival curves, patients with tumour stage I-II were associated with longer OS $(p=0.005)$ compared to patients with tumour stage III-IV. Moreover, well differentiated tumours were also associated with longer OS $(p=0.029)$ compared to poorly differentiated ones (Fig. 1).

\section{Immune cell number and patient survival}

In the HNSCC surgical specimens, immune cells were detected by using specific antibodies against CD8, FoxP3 and CD68 within the ST and the IT compartments (Fig. 2a-c). Lymphocytes T (CD8+), Treg (FoxP3+) and macrophages (CD68+) were counted in 5 random fields (magnification 400x) in both ST and IT compartments (Fig. 2d-i).

Cut-offs were calculated using RStudio software regarding optimal HR and $p$-values for OS and patients were classified as expressing a low or high number of immune cells. The cut-off values were 55.8 cells (CD8, ST), 53.55 cells (CD8, IT), 59.5 cells (FoxP3, ST), 11.5 cells (FoxP3, IT), 143.5 cells (CD68, ST) and 22.1 cells (CD68, IT). Additionally, univariate and multivariate analyses (Cox regression) were performed 
for the three immuno-markers CD8, FoxP3 and CD68 in the two compartments for RFS and OS (Table 2). Multivariate analysis showed that the CD8 + cell number was a strong and independent prognostic marker.

Table 2

Univariate and multivariate Cox regression models evaluating the influence of stromal (ST) or intra-tumoral (IT) of CD8, FoxP3 and CD68 on RFS and OS. 0 and 1 are related to the cut-off.

\begin{tabular}{|c|c|c|c|c|}
\hline \multirow[t]{2}{*}{ Univariate analysis } & \multicolumn{2}{|c|}{ Relapse-free survival } & \multicolumn{2}{|c|}{ Overall survival } \\
\hline & $p$-value & $\mathrm{HR}(95 \% \mathrm{Cl})$ & $p$-value & $\mathrm{HR}(95 \% \mathrm{Cl})$ \\
\hline CD8 ST 0-1 & 0.958 & $0.98(0.48-2.02)$ & 0.026 & $3.19(1.15-8.90)$ \\
\hline CD8 IT 1 - 0 & 0.011 & $3.73(1.35-10.31)$ & 0.025 & $3.20(1.16-8.84)$ \\
\hline FoxP3 ST 1 - 0 & 0.002 & $1.97(1.28-3.04)$ & 0.002 & $1.95(1.29-2.96)$ \\
\hline FoxP3 IT 1 - 0 & 0.008 & $1.84(1.17-2.87)$ & 0.001 & $2.16(1.36-3.41)$ \\
\hline CD68 ST 1 - 0 & 0.206 & $1.56(0.78-3.11)$ & 0.076 & $2.12(0.93-4.85)$ \\
\hline CD68 IT 0-1 & 0.004 & $1.86(1.21-2.85)$ & 0.005 & $1.79(1.19-2.69)$ \\
\hline \multirow[t]{2}{*}{ Multivariate analysis } & \multicolumn{2}{|c|}{ Relapse-free survival } & \multicolumn{2}{|c|}{ Overall survival } \\
\hline & $p$-value & $\mathrm{HR}(95 \% \mathrm{Cl})$ & $p$-value & $\mathrm{HR}(95 \% \mathrm{Cl})$ \\
\hline CD8 ST 0-1 & 0.847 & $1.10(0.43-2.78)$ & 0.028 & $5.03(1.19-21.31)$ \\
\hline CD8 IT 1 - 0 & 0.024 & $3.36(1.17-9.67)$ & 0.038 & $3.08(1.06-8.94)$ \\
\hline FoxP3 ST 1 - 0 & 0.255 & $1.87(0.64-5.49)$ & 0.147 & $2.46(0.73-8.28)$ \\
\hline FoxP3 IT 1 - 0 & 0.056 & $1.88(0.98-3.57)$ & 0.109 & $1.63(0.90-2.98)$ \\
\hline CD68 ST 1 - 0 & 0.13 & $1.63(0.86-3.09)$ & 0.961 & $1.02(0.56-1.83)$ \\
\hline CD68 IT 0-1 & 0.6 & $1.22(0.58-2.55)$ & 0.080 & $1.98(0.92-4.27)$ \\
\hline
\end{tabular}


Table 3

Univariate and multivariate Cox regression models

evaluating the tumour stage, the histological grade and the immunoscore and the OS.

\begin{tabular}{|lll|}
\hline Univariate analysis & \multicolumn{2}{l|}{ Overall survival } \\
\hline & $p$-value & $\mathrm{HR}(95 \% \mathrm{Cl})$ \\
\hline Tumour stage & $\mathbf{0 . 0 0 5}$ & $1.91(1.22-3.00)$ \\
\hline Histological grade & $\mathbf{0 . 0 2 9}$ & $0.62(0.40-0.95)$ \\
\hline ImmuneScore & $\mathbf{0 . 0 0 2}$ & $9.87(2.38-40.99)$ \\
\hline Multivariate analysis & Overall survival \\
\hline & $p$-value & $\mathrm{HR}(95 \% \mathrm{Cl})$ \\
\hline Tumour stage & 0.373 & $1.36(0.69-2.69)$ \\
\hline Histological grade & 0.401 & $1.31(0.70-2.46)$ \\
\hline ImmuneScore & $\mathbf{0 . 0 1 8}$ & $11.17(1.52-82.12)$ \\
\hline
\end{tabular}

Using these cut-offs, Kaplan-Meier curves were established for each immune cell in each compartment for RFS and OS. Regarding the ST compartment, longer RFS was significantly associated with a high FoxP3 + cell number, while longer OS correlated with low CD8 + and high FoxP3 + cell numbers (Fig. 3). In $\mathrm{ST}$, the CD68 + cell number did not correlate with RFS or OS. In the IT compartment, low CD8+, high FoxP3 + and low CD68 + cell numbers were significantly linked to a longer RFS as well as a longer OS (Fig. 4).

\section{Immunoscore and patient survival}

The Fig. 5a describes how we calculated our immunoscore. Each tumour of the patients was categorized into low (Lo) or high (Hi) density for each immune cell in each tumour region according to the cut-off values. Depending on the type of immune cells and the tumour compartment, the Lo and Hi classes were associated to the blue and red groups corresponding to the 0 and 1 scores, respectively. The immunoscore was created by adding the individual score $(0 / 1)$ of each marker, which was significant for OS. Based on univariate analyses (Table 1), we included CD8 ST/IT, FoxP3 ST/IT and CD68 IT in the immunoscore. CD68 + cell number in the ST did not correlate with RFS or OS and will therefore not included in the immunoscore. Thus, the scoring system ranges from 0 to 5 . Kaplan-Meier curves were drawn for each value of the immunoscore and a cut-off discriminating good and poor patient prognosis was chosen at 3 (Supplementary Fig. 1). Thus, each patient was classified in the blue group (good prognosis, low immune score $<3, n=23$ ) or in the red group (poor prognosis, high immune score $>3, n=$ 97). 
Very significant differences were observed between the blue and red immunoscore. Kaplan-Meier curves using our immunoscore showed a significant correlation for RFS $(p=0.007)$ and OS $(p=0.002)$ (Figs. 5b and $c$ respectively) with high immunoscore $(>3)$ being associated with shorter RFS and OS, compared with low immunoscore $(<3)$ which was associated with better prognosis for RFS and OS.

Finally, we performed univariate and multivariate Cox regression analyses to compare our immunoscore with the conventional tumour stage and histological grade. Our immunoscore correlated more significantly, and with a greater separation of the two groups, regarding OS $(p=0.002, H R=9.87)$ compared to tumour stage $(p=0.005, H R=1.91)$ and histological grade $(p=0.029, H R=0.62)($ Table 2$)$. Multivariate analyses revealed that the immunoscore was the only parameter associated with a strong and independent prognosis value.

\section{Discussion}

To the best of our knowledge, this study assessed, for the first time, the abundance and distribution of innate and adaptive cellular elements according to CD8 T cells, FoxP3 Treg and CD68 macrophages in a series of 258 patients with HNSCC in order to define a more global immune contexture. Then, we investigated their potential prognostic value separately and in combination to stratify patients using a low immunoscore corresponding to a longer RFS and OS, whereas a high immunoscore was related to a poorer prognosis. Our results confirm that the establishment of an immunoscore has a higher prognostic value than that of TNM staging system and histological grade.

For many years, clinical research around head and neck cancers has been constantly asking for new prognostic biomarkers to better guide patient management. Given the complexity of the interactions between immune infiltrates and the TME, the tumour must no longer be considered as a single entity but must be studied in relation to its microenvironment and the host immune response in order to bring clinical relevance and value in determining the tumour progression and the patient prognosis. As such, many clinicians and researchers have been interested in the infiltration of immune cells in head and neck cancers. In most cases, massive infiltration of CD8 + T lymphocytes in HPV- as well as in HPV + oropharyngeal carcinomas correlates positively with patient prognosis (Nordfors et al. 2013; Oguejiofor et al. 2015; Ward et al. 2014; Sivars et al. 2017; Poropatich et al. 2017). CD8 + TILs have also been established as an independent prognostic marker in patients diagnosed with oropharyngeal squamous cell carcinoma (De Meulenaere et al. 2017). Recently, Echarti et al. confirmed these findings by quantifying CD8 + and FoxP3 + T lymphocytes in epithelial and ST compartments (Echarti et al. 2019). The relationship between tumour-infiltrating Treg and patient prognosis has been evaluated in many malignancies and associated with different prognostic response according to the sites of primary cancer. The prognostic significance of FoxP3 + Tregs has been extensively studied (Shang et al. 2015) and high Treg cell recruitment is reported to correlated with poor prognosis in breast, liver, pancreatic, ovarian, cervical and renal cancer (Merlo et al. 2009; Fu et al. 2007; Hiraoka et al. 2006; Curiel et al. 2004; Shah et al. 2011; Siddiqui et al. 2007) whereas it may also correlate with longer survival as demonstrated in colorectal, bladder cancer, HNSCC and lymphoma (Seminerio et al. 2019; Salama et al. 2009; Winerdal et 
al. 2011; Badoual et al. 2006; Tzankov et al. 2008). The meta-analysis of Shang et al. compares 76 datasets which highlight the prognostic role of FoxP3 + in 17 cancer types (Shang et al. 2015). For HNSCC, Treg infiltration is highly controversial with conflicting results. While several studies underline the deleterious impact of massive infiltration by Tregs on prognosis, we and others have already showed the opposite (Badoual et al. 2006; Bron et al. 2013). Indeed, we previously reported that FoxP3 + infiltration was associated with longer RFS and OS of patients suffering from HNSSC (Seminerio et al. 2019; Kindt et al. 2017; Shang et al. 2015). The concomitance which is often observed between Treg and cytotoxic $T$ infiltrates could tip the balance towards a favourable immune orientation and consequently participate in the good paradoxical prognostic value of this population. In addition, HNSCC are heterogeneous tumours located in certain anatomical sites rich in lymphoid tissue such as oropharynx, which may explain a greater recruitment of Treg in this site. Importantly, a crucial point of the debate was elucidated in 2016 when Saito et al. demonstrated the existence of two populations of FoxP3 + Tregs in colorectal cancers (Saito et al. 2016). The first one was the immunosuppressive FoxP $3^{\text {high }}$-expressing cells classically associated with a poor prognosis and the second was characterized by non-suppressive capacities and the absence of expression of CD45 (FoxP3 ${ }^{\text {low }}$ CD45RA-), the naïve T cell marker. These two populations are significantly correlated with opposing prognoses in colorectal cancer. While the first is associated with a poor prognosis, the infiltration of FoxP3 ${ }^{\text {low }}$, which secrete pro-inflammatory cytokines such as IL12 and TGF $\beta$, is characteristic of better patient survival (Saito et al. 2016). In addition, we suggest another hypothesis that could explain the infiltration of FoxP3 + Tregs in head and neck cancers. Indeed, they grow in a septic environment in contact with a resident microbiota, just like colon cancers which are also associated with a better prognosis when the number of Treg is high. This microbiota interacts and may modulate the host oral immune cells and such alterations in Treg functions have already been observed in oral infections (Ladoire, Martin, and Ghiringhelli 2011; Pandiyan et al. 2019). Moreover, studies demonstrated the protective effects of oral FoxP3 + Treg in some local infections (Yamazaki et al. 2012). Given the crosstalk between these immunosuppressive regulatory cells and the cytotoxic lymphocytes, which are the anticancer mediators of the immune system, it seems crucial to consider these two entities in combination in order to better understand the reasons for the contradictory results reported in the literature.

Macrophages also constitute an important partner in innate and adaptive inflammatory responses. Beyond the binary classification of pro-inflammatory M1 and anti-inflammatory M2 macrophages, it is now accepted that these two phenotypes are only the extremes of a continuum of polarization, in which there is a spectrum of differentiated macrophages (Palma et al. 2018). Among these differentiated macrophages, TAMs are involved in immune tolerance, inflammatory disease and cancer (Aras and Zaidi 2017) and are considered as pro-tumorigenic immune cells. They stimulate Treg differentiation and secrete several factors (e.g. TGF $\beta$, TNFa and IL-10) to create a favourable environment for tumour progression and to inhibit the anti-tumour effects of immune cells (Jérôme R. Lechien et al. 2020). Regarding prognosis, tumour infiltration by TAMs is reported to be an unfavourable parameter for patient survival (Esch et al. 2015; Davis, Van Waes, and Allen 2016; Costa et al. 2013). Our recent study has shown that a high recruitment of CD68 + macrophages in a population of 110 HNSCC was correlated with 
shorter patient RFS and OS. Moreover, the analysis of the M1/M2 ratio in the TME, with a double staining using anti-CD68/anti-CD163 antibodies, revealed that $80 \%$ of the macrophage population had an M2 phenotype (Seminerio et al. 2018). Interestingly, it appears that TAMs can secrete IL-10 in order to induce the differentiation of T lymphocytes into Treg (Murai et al. 2009) and thus participate in immune cell evasion.

The existence of complex regulatory loops between these three major mediators of the immune system has led us to quantify their recruitment in ST and IT localizations in a large cohort of head and neck tumours. Indeed, we hypothesized that analysing each tumour compartment (ST versus IT) may provide distinct and complementary prognostic information. This was also supported in the context of rectal cancer where the location of CD8 + T cells and FoxP3 + Treg cells in distinct compartments (epithelium versus stroma) result in different prognostic responses (Posselt et al. 2016). Combining the three markers in an immunoscore signature, we found that a low immunoscore was significantly associated with a longer RFS and a prolonged OS. Based on the calculated optimal cut-offs, the IT immune infiltrations associated with a better prognosis correspond to a high number of CD8 and FoxP3 and a low number of CD68 macrophages. Conversely, in the ST, a better prognosis was observed in patients with a low CD8 infiltration but always a high number of FoxP3. Because of the tumour lysis capacity of CD8 cells, these anticancer actors are strong allies for cancer patients. On the other hand, despite their anti-tumour response suppressor characteristics, Tregs infiltrates have been found to be associated with a favourable outcome, which may be partially attributed to a downregulation of the inflammatory process (Shang et al. 2015; Badoual et al. 2006). A correlation had also been found between a higher number of Treg in the stroma and an absence of metastatic lymph nodes which means that Treg could generate proinflammatory processes in the tumour microenvironment favouring a delay in the tumour evolution and consequently would generate a better prognosis of the patients (Bron et al. 2013). Furthermore, Khoury et al. recently proposed that IT TILs were distinct from ST TILs in their biological behaviour (Khoury et al. 2018). Indeed, ST is constituted of many components which can impair host immune responses, such as fibroblasts, macrophages, or endothelial cells, underlining the difference in CD8 TILs located in the ST from CD8 + TILs within tumour cells.

Finally, our multivariate analysis, evaluating the tumour stage, the histological grade and the immunoscore regarding to OS, revealed an impressive HR for immunoscore which can significantly predict the OS of patients. By contrast, in head and neck cancer, only a few studies have identified an immunoscore positively correlating with RFS and OS. In two studies, immunoscore only included TILs, and more precisely CD3 + and CD8 + cells (Lechner et al. 2017; Zhang et al. 2018). A recent study confirmed the positive prognostic impact of TILs in oral squamous cell carcinomas by increasing the immune signature to seven markers that can predict patient survival (Zhou et al. 2020).

\section{Declarations}

\section{Funding:}


This study was funded by the University of Mons, ProtherWal and the Walloon Region and FRMH. S.F. is funded by the University of Mons and the Epicura Hospital and G.D. is funded by the Walloon Region via the ProtherWal society.

\section{Conflicts of interest:}

The authors declare that they have no conflict of interest.

\section{Availability of data and material:}

The datasets generated during and/or analysed during the current study are available from the corresponding author on reasonable request.

\section{Code availability:}

Not applicable

\section{Authors' contributions:}

Sonia Furgiuele, Géraldine Descamps, Fabrice Journe, and Sven Saussez contributed to the study conception and design. Material preparation, data collection and analysis were performed by Sonia Furgiuele, Géraldine Descamps, and Fabrice Journe. The first draft of the manuscript was written by Sonia Furgiuele and Géraldine Descamps and all authors commented on previous versions of the manuscript. All authors read and approved the final manuscript.

\section{Ethics approval:}

Ethical approval was waived by the Institutional Review Board of Jules Bordet Institute (number CE2319) in view of the retrospective nature of the study and all the procedures being performed were part of the routine care.

\section{Consent to participate:}

Informed consent was obtained from all individual participants included in the study.

\section{Consent for publication:}

Not applicable

\section{Acknowledgments:}

We thank Saint-Pierre Hospital (Brussels, Belgium), Jules Bordet Institute (Brussels, Belgium), EpiCURA Baudour Hospital (Baudour, Belgium) and CHU Sart-Tilman (Liège, Belgium) for FFPE specimens and clinical data. We also thank UMONS, ProtherWal, the Walloon Region and FRMH for funding this work. 


\section{References}

1. Aras, Shweta, and M Raza Zaidi. 2017. "TAMeless Traitors: Macrophages in Cancer Progression and Metastasis." British Journal of Cancer 117 (11): 1583-1591. doi:10.1038/bjc.2017.356.

2. Badoual, Cécile, Stéphane Hans, José Rodriguez, Severine Peyrard, Christophe Klein, Nour El Houda Agueznay, Véronique Mosseri, et al. 2006. "Prognostic Value of Tumor-Infiltrating CD4+ T-Cell Subpopulations in Head and Neck Cancers." Clinical Cancer Research 12 (2): 465-472. doi:10.1158/1078-0432.CCR-05-1886.

3. Bron, Luc, Camilla Jandus, Snezana Andrejevic-Blant, Daniel E. Speiser, Philippe Monnier, Pedro Romero, and Jean-Paul Rivals. 2013. "Prognostic Value of Arginase-II Expression and Regulatory TCell Infiltration in Head and Neck Squamous Cell Carcinoma." International Journal of Cancer 132 (3): E85-E93. doi:10.1002/ijc.27728.

4. Canning, Madison, Gang Guo, Miao Yu, Calvin Myint, Michael W. Groves, James Kenneth Byrd, and Yan Cui. 2019. "Heterogeneity of the Head and Neck Squamous Cell Carcinoma Immune Landscape and Its Impact on Immunotherapy." Frontiers in Cell and Developmental Biology 7: 52. doi:10.3389/fcell.2019.00052.

5. Chen, Hong, Bairong Xia, Tongsen Zheng, and Ge Lou. 2020. "Immunoscore System Combining CD8 and PD-1/PD-L1: A Novel Approach That Predicts the Clinical Outcomes for Cervical Cancer." The International Journal of Biological Markers 35 (1): 65-73. doi:10.1177/1724600819888771.

6. Chen, Samantha M. Y., Alexandra L. Krinsky, Rachel A. Woolaver, Xiaoguang Wang, Zhangguo Chen, and Jing H. Wang. 2020. "Tumor Immune Microenvironment in Head and Neck Cancers." Molecular Carcinogenesis, February. doi:10.1002/mc.23162.

7. Costa, Nadia Lago, Marize Campos Valadares, Pedro Paulo Chaves Souza, Elismauro Francisco Mendonça, José Carlos Oliveira, Tarcíla Aparecida Silva, and Aline Carvalho Batista. 2013. "TumorAssociated Macrophages and the Profile of Inflammatory Cytokines in Oral Squamous Cell Carcinoma." Oral Oncology 49 (3): 216-223. doi:10.1016/j.oraloncology.2012.09.012.

8. Curiel, Tyler J., George Coukos, Linhua Zou, Xavier Alvarez, Pui Cheng, Peter Mottram, Melina Evdemon-Hogan, et al. 2004. "Specific Recruitment of Regulatory T Cells in Ovarian Carcinoma Fosters Immune Privilege and Predicts Reduced Survival." Nature Medicine 10 (9): 942-949. doi:10.1038/nm1093.

9. Davis, Ruth J., Carter Van Waes, and Clint T. Allen. 2016. "Overcoming Barriers to Effective Immunotherapy: MDSCs, TAMs, and Tregs as Mediators of the Immunosuppressive Microenvironment in Head and Neck Cancer." Oral Oncology 58 (July): 59-70. doi:10.1016/j.oraloncology.2016.05.002.

10. De Meulenaere, Astrid, Tijl Vermassen, Sandrine Aspeslagh, Philippe Deron, Fréderic Duprez, Debby Laukens, Jo Van Dorpe, Liesbeth Ferdinande, and Sylvie Rottey. 2017. "Tumor PD-L1 Status and CD8+ Tumor-Infiltrating T Cells: Markers of Improved Prognosis in Oropharyngeal Cancer." Oncotarget 8 (46): 80443-80452. doi:10.18632/oncotarget.19045. 
11. de Ruiter, Emma J., Marc L. Ooft, Lot A. Devriese, and Stefan M. Willems. 2017. "The Prognostic Role of Tumor Infiltrating T-Lymphocytes in Squamous Cell Carcinoma of the Head and Neck: A Systematic Review and Meta-Analysis." Oncoimmunology 6 (11). doi:10.1080/2162402X.2017.1356148.

12. Echarti, Alessia, Markus Hecht, Maike Büttner-Herold, Marlen Haderlein, Arndt Hartmann, Rainer Fietkau, and Luitpold Distel. 2019. "CD8+ and Regulatory T Cells Differentiate Tumor Immune Phenotypes and Predict Survival in Locally Advanced Head and Neck Cancer." Cancers 11 (9). doi:10.3390/cancers11091398.

13. Esch, Edith M. G. van, Mariette I. E. van Poelgeest, J. Baptist M. Z. Trimbos, Gert Jan Fleuren, Ekaterina S. Jordanova, and Sjoerd H. van der Burg. 2015. "Intraepithelial Macrophage Infiltration Is Related to a High Number of Regulatory T Cells and Promotes a Progressive Course of HPV-Induced Vulvar Neoplasia." International Journal of Cancer 136 (4): E85-E94. doi:10.1002/ijc.29173.

14. Evrard, Diane, Petr Szturz, Annemilaï Tijeras-Raballand, Lucile Astorgues-Xerri, Chloé Abitbol, Valérie Paradis, Eric Raymond, Sébastien Albert, Béatrix Barry, and Sandrine Faivre. 2019. "Macrophages in the Microenvironment of Head and Neck Cancer: Potential Targets for Cancer Therapy." Oral Oncology 88: 29-38. doi:10.1016/j.oraloncology.2018.10.040.

15. Ferlay, J., M. Colombet, I. Soerjomataram, C. Mathers, D. M. Parkin, M. Piñeros, A. Znaor, and F. Bray. 2019. "Estimating the Global Cancer Incidence and Mortality in 2018: GLOBOCAN Sources and Methods." International Journal of Cancer 144 (8): 1941-1953. doi:10.1002/ijc.31937.

16. Fu, Junliang, Dongping Xu, Zhenwen Liu, Ming Shi, Ping Zhao, Baoyun Fu, Zheng Zhang, et al. 2007. "Increased Regulatory T Cells Correlate with CD8 T-Cell Impairment and Poor Survival in Hepatocellular Carcinoma Patients." Gastroenterology 132 (7): 2328-2339. doi:10.1053/j.gastro.2007.03.102.

17. Gao, Jiani, Yijiu Ren, Haoyue Guo, Rui Mao, Huikang Xie, Hang Su, Yunlang She, et al. 2020. "A New Method for Predicting Survival in Stage I Non-Small Cell Lung Cancer Patients: Nomogram Based on Macrophage Immunoscore, TNM Stage and Lymphocyte-to-Monocyte Ratio." Annals of Translational Medicine 8 (7). doi:10.21037/atm.2020.03.113.

18. Gavrielatou, Niki, Stergios Doumas, Panagiota Economopoulou, Periklis G. Foukas, and Amanda Psyrri. 2020. "Biomarkers for Immunotherapy Response in Head and Neck Cancer." Cancer Treatment Reviews 84 (March): 101977. doi:10.1016/j.ctrv.2020.101977.

19. Hiraoka, Nobuyoshi, Kaoru Onozato, Tomoo Kosuge, and Setsuo Hirohashi. 2006. "Prevalence of FOXP3+ Regulatory T Cells Increases during the Progression of Pancreatic Ductal Adenocarcinoma and Its Premalignant Lesions." Clinical Cancer Research: An Official Journal of the American Association for Cancer Research 12 (18): 5423-5434. doi:10.1158/1078-0432.CCR-06-0369.

20. Jiang, Yuming, Qi Zhang, Yanfeng Hu, Tuanjie Li, Jiang Yu, Liying Zhao, Gengtai Ye, et al. 2018. "ImmunoScore Signature: A Prognostic and Predictive Tool in Gastric Cancer." Annals of Surgery 267 (3): 504-513. doi:10.1097/SLA.0000000000002116. 
21. Khoury, Thaer, Vidya Nagrale, Mateusz Opyrchal, Xuan Peng, Dan Wang, and Song Yao. 2018. "Prognostic Significance of Stromal Versus Intratumoral Infiltrating Lymphocytes in Different Subtypes of Breast Cancer Treated with Cytotoxic Neoadjuvant Chemotherapy." Applied Immunohistochemistry \& Molecular Morphology: AIMM 26 (8): 523-532. doi:10.1097/PAl.0000000000000466.

22. Kindt, Nadège, Géraldine Descamps, Imelda Seminerio, Justine Bellier, Jérôme R. Lechien, Quentin Mat, Charles Pottier, Philippe Delvenne, Fabrice Journé, and Sven Saussez. 2017. "High Stromal Foxp3-Positive T Cell Number Combined to Tumor Stage Improved Prognosis in Head and Neck Squamous Cell Carcinoma." Oral Oncology 67 (April): 183-191. doi:10.1016/j.oraloncology.2017.02.023.

23. Kindt, Nadège, Géraldine Descamps, Imelda Seminerio, Justine Bellier, Jérôme R. Lechien, Charles Pottier, Denis Larsimont, Fabrice Journé, Philippe Delvenne, and Sven Saussez. 2016. “Langerhans Cell Number Is a Strong and Independent Prognostic Factor for Head and Neck Squamous Cell Carcinomas." Oral Oncology 62 (November): 1-10. doi:10.1016/j.oraloncology.2016.08.016.

24. Ladoire, Sylvain, François Martin, and François Ghiringhelli. 2011. "Prognostic Role of FOXP3+ Regulatory T Cells Infiltrating Human Carcinomas: The Paradox of Colorectal Cancer." Cancer Immunology, Immunotherapy: Cll 60 (7): 909-918. doi:10.1007/s00262-011-1046-y.

25. Lechien, Jérôme R., Géraldine Descamps, Imelda Seminerio, Sonia Furgiuele, Didier Dequanter, Francois Mouawad, Cécile Badoual, Fabrice Journe, and Sven Saussez. 2020. "HPV Involvement in the Tumor Microenvironment and Immune Treatment in Head and Neck Squamous Cell Carcinomas." Cancers 12 (5). doi:10.3390/cancers 12051060.

26. Lechien, Jerome R., Imelda Seminerio, Géraldine Descamps, Quentin Mat, Francois Mouawad, Stéphane Hans, Morbize Julieron, et al. 2019. "Impact of HPV Infection on the Immune System in Oropharyngeal and Non-Oropharyngeal Squamous Cell Carcinoma: A Systematic Review." Cells 8 (9). doi:10.3390/cells8091061.

27. Lechner, Axel, Hans Schlößer, Sacha I. Rothschild, Martin Thelen, Sabrina Reuter, Peter Zentis, Alexander Shimabukuro-Vornhagen, et al. 2017. "Characterization of Tumor-Associated TLymphocyte Subsets and Immune Checkpoint Molecules in Head and Neck Squamous Cell Carcinoma." Oncotarget 8 (27): 44418-44433. doi:10.18632/oncotarget.17901.

28. Leemans, C. René, Peter J. F. Snijders, and Ruud H. Brakenhoff. 2018. "The Molecular Landscape of Head and Neck Cancer." Nature Reviews Cancer 18 (5): 269-282. doi:10.1038/nrc.2018.11.

29. Lyford-Pike, Sofia, Shiwen Peng, Geoffrey D. Young, Janis M. Taube, William H. Westra, Belinda Akpeng, Tullia C. Bruno, et al. 2013. "Evidence for a Role of the PD-1:PD-L1 Pathway in Immune Resistance of HPV-Associated Head and Neck Squamous Cell Carcinoma." Cancer Research 73 (6): 1733-1741. doi:10.1158/0008-5472.CAN-12-2384.

30. Merlo, Andrea, Patrizia Casalini, Maria Luisa Carcangiu, Chiara Malventano, Tiziana Triulzi, Sylvie Mènard, Elda Tagliabue, and Andrea Balsari. 2009. "FOXP3 Expression and Overall Survival in Breast 
Cancer." Journal of Clinical Oncology: Official Journal of the American Society of Clinical Oncology 27 (11): 1746-1752. doi:10.1200/JC0.2008.17.9036.

31. Mirza, Adal H., Gareth Thomas, Christian H. Ottensmeier, and Emma V. King. 2019. "Importance of the Immune System in Head and Neck Cancer." Head \& Neck 41 (8): 2789-2800. doi:10.1002/hed.25716.

32. Murai, Masako, Olga Turovskaya, Gisen Kim, Rajat Madan, Christopher L Karp, Hilde Cheroutre, and Mitchell Kronenberg. 2009. "Interleukin 10 Acts on Regulatory T Cells to Maintain Expression of the Transcription Factor Foxp3 and Suppressive Function in Mice with Colitis." Nature Immunology 10 (11): 1178-1184. doi:10.1038/ni.1791.

33. Nie, Run-Cong, Shu-Qiang Yuan, Yun Wang, Ying-Bo Chen, Yan-Yu Cai, Shi Chen, Shu-Man Li, et al. 2019. "Robust Immunoscore Model to Predict the Response to Anti-PD1 Therapy in Melanoma." Aging (Albany NY) 11 (23): 11576-11590. doi:10.18632/aging.102556.

34. Nordfors, Cecilia, Nathalie Grün, Nikolaos Tertipis, Andreas Ährlund-Richter, Linnea Haeggblom, Lars Sivars, Juan Du, et al. 2013. "CD8+ and CD4+ Tumour Infiltrating Lymphocytes in Relation to Human Papillomavirus Status and Clinical Outcome in Tonsillar and Base of Tongue Squamous Cell Carcinoma." European Journal of Cancer 49 (11): 2522-2530. doi:10.1016/j.ejca.2013.03.019.

35. Oguejiofor, K, J Hall, C Slater, G Betts, G Hall, N Slevin, S Dovedi, P L Stern, and C M L West. 2015. "Stromal Infiltration of CD8 T Cells Is Associated with Improved Clinical Outcome in HPV-Positive Oropharyngeal Squamous Carcinoma." British Journal of Cancer 113 (6): 886-893. doi:10.1038/bjc.2015.277.

36. Pagès, Franck, Bernhard Mlecnik, Florence Marliot, Gabriela Bindea, Fang-Shu Ou, Carlo Bifulco, Alessandro Lugli, et al. 2018. "International Validation of the Consensus Immunoscore for the Classification of Colon Cancer: A Prognostic and Accuracy Study." Lancet (London, England) 391 (10135): 2128-2139. doi:10.1016/S0140-6736(18)30789-X.

37. Palma, Alessandro, Abdul Salam Jarrah, Paolo Tieri, Gianni Cesareni, and Filippo Castiglione. 2018. "Gene Regulatory Network Modeling of Macrophage Differentiation Corroborates the Continuum Hypothesis of Polarization States." Frontiers in Physiology 9 (November). doi:10.3389/fphys.2018.01659.

38. Pandiyan, Pushpa, Natarajan Bhaskaran, Mangge Zou, Elizabeth Schneider, Sangeetha Jayaraman, and Jochen Huehn. 2019. "Microbiome Dependent Regulation of Tregs and Th17 Cells in Mucosa." Frontiers in Immunology 10. Frontiers. doi:10.3389/fimmu.2019.00426.

39. Petrizzo, Annacarmen, and Luigi Buonaguro. 2016. "Application of the Immunoscore as Prognostic Tool for Hepatocellular Carcinoma." Journal for Immunotherapy of Cancer 4 (November). doi:10.1186/s40425-016-0182-5.

40. Poropatich, Kate, David Hernandez, Joel Fontanarosa, Koshonna Brown, Gayle Woloschak, Ajit Paintal, Kirtee Raparia, and Sandeep Samant. 2017. "Peritumoral Cuffing by T-Cell Tumor-Infiltrating Lymphocytes Distinguishes HPV-Related Oropharyngeal Squamous Cell Carcinoma from Oral Cavity 
Squamous Cell Carcinoma." Journal of Oral Pathology and Medicine 46 (10): 972-978. doi:10.1111/jop.12605.

41. Posselt, Rebecca, Katharina Erlenbach-Wünsch, Matthias Haas, Jonas Je $\Delta$ berger, Maike BüttnerHerold, Marlen Haderlein, Markus Hecht, Arndt Hartmann, Rainer Fietkau, and Luitpold V. Distel. 2016. "Spatial Distribution of FoxP3+ and CD8+ Tumour Infiltrating T Cells Reflects Their Functional Activity." Oncotarget 7 (37): 60383-60394. doi:10.18632/oncotarget.11039.

42. Saito, Takuro, Hiroyoshi Nishikawa, Hisashi Wada, Yuji Nagano, Daisuke Sugiyama, Koji Atarashi, Yuka Maeda, et al. 2016. "Two FOXP3(+)CD4(+) T Cell Subpopulations Distinctly Control the Prognosis of Colorectal Cancers." Nature Medicine 22 (6): 679-684. doi:10.1038/nm.4086.

43. Sakaguchi, Shimon, Makoto Miyara, Cristina M. Costantino, and David A. Hafler. 2010. "FOXP3 + Regulatory T Cells in the Human Immune System." Nature Reviews Immunology 10 (7): 490-500. doi:10.1038/nri2785.

44. Salama, Paul, Michael Phillips, Fabienne Grieu, Melinda Morris, Nik Zeps, David Joseph, Cameron Platell, and Barry lacopetta. 2009. "Tumor-Infiltrating FOXP3+ T Regulatory Cells Show Strong Prognostic Significance in Colorectal Cancer." Journal of Clinical Oncology: Official Journal of the American Society of Clinical Oncology 27 (2): 186-192. doi:10.1200/JCO.2008.18.7229.

45. Seminerio, Imelda, Géraldine Descamps, Sophie Dupont, Lisa de Marrez, Jean-Alexandre Laigle, Jérôme R. Lechien, Nadège Kindt, Fabrice Journe, and Sven Saussez. 2019. "Infiltration of FoxP3+ Regulatory T Cells Is a Strong and Independent Prognostic Factor in Head and Neck Squamous Cell Carcinoma." Cancers 11 (2). doi:10.3390/cancers11020227.

46. Seminerio, Imelda, Nadège Kindt, Géraldine Descamps, Justine Bellier, Jérôme R. Lechien, Quentin Mat, Charles Pottier, Fabrice Journé, and Sven Saussez. 2018. "High Infiltration of CD68+ Macrophages Is Associated with Poor Prognoses of Head and Neck Squamous Cell Carcinoma Patients and Is Influenced by Human Papillomavirus." Oncotarget 9 (13): 11046-11059. doi:10.18632/oncotarget.24306.

47. Shah, Walayat, Xiaofei Yan, Li Jing, Yi Zhou, Hongwei Chen, and Yili Wang. 2011. "A Reversed CD4/CD8 Ratio of Tumor-Infiltrating Lymphocytes and a High Percentage of CD4+FOXP3+ Regulatory T Cells Are Significantly Associated with Clinical Outcome in Squamous Cell Carcinoma of the Cervix." Cellular and Molecular Immunology 8 (1): 59-66. doi:10.1038/cmi.2010.56.

48. Shang, Bin, Yao Liu, Shu-juan Jiang, and Yi Liu. 2015. "Prognostic Value of Tumor-Infiltrating FoxP3+ Regulatory T Cells in Cancers: A Systematic Review and Meta-Analysis." Scientific Reports 5 (October). doi:10.1038/srep15179.

49. Siddiqui, Sameer A., Xavier Frigola, Sandra Bonne-Annee, Maria Mercader, Susan M. Kuntz, Amy E. Krambeck, Shomik Sengupta, et al. 2007. "Tumor-Infiltrating Foxp3-CD4+CD25+ T Cells Predict Poor Survival in Renal Cell Carcinoma." Clinical Cancer Research: An Official Journal of the American Association for Cancer Research 13 (7): 2075-2081. doi:10.1158/1078-0432.CCR-06-2139.

50. Sivars, Lars, David Landin, Nathalie Grün, Andrea Vlastos, Linda Marklund, Sushma Nordemar, Torbjörn Ramqvist, Eva Munck-Wikland, Anders Näsman, and Tina Dalianis. 2017. "Validation of 
Human Papillomavirus as a Favourable Prognostic Marker and Analysis of CD8+ Tumour-Infiltrating Lymphocytes and Other Biomarkers in Cancer of Unknown Primary in the Head and Neck Region." Anticancer Research 37 (2): 665-673.

51. Tahkola, Kyösti, Jukka-Pekka Mecklin, Erkki-Ville Wirta, Maarit Ahtiainen, Olli Helminen, Jan Böhm, and IImo Kellokumpu. 2018. "High Immune Cell Score Predicts Improved Survival in Pancreatic Cancer." Virchows Archiv: An International Journal of Pathology 472 (4): 653-665. doi:10.1007/s00428-018-2297-1.

52. Tzankov, Alexandar, Cecile Meier, Petra Hirschmann, Philip Went, Stefano A. Pileri, and Stephan Dirnhofer. 2008. "Correlation of High Numbers of Intratumoral FOXP3+ Regulatory T Cells with Improved Survival in Germinal Center-like Diffuse Large B-Cell Lymphoma, Follicular Lymphoma and Classical Hodgkin's Lymphoma." Haematologica 93 (2): 193-200. doi:10.3324/haematol.11702.

53. Valkenburg, Kenneth C., Amber E. de Groot, and Kenneth C. Pienta. 2018. "Targeting the Tumour Stroma to Improve Cancer Therapy." Nature Reviews. Clinical Oncology 15 (6): 366-381. doi:10.1038/s41571-018-0007-1.

54. Ward, M J, S M Thirdborough, T Mellows, C Riley, S Harris, K Suchak, A Webb, et al. 2014. "TumourInfiltrating Lymphocytes Predict for Outcome in HPV-Positive Oropharyngeal Cancer." British Journal of Cancer 110 (2): 489-500. doi:10.1038/bjc.2013.639.

55. Windon, Melina J., Gypsyamber D'Souza, Eleni M. Rettig, William H. Westra, Annemieke van Zante, Steven J. Wang, William R. Ryan, et al. 2018. “INCREASING PREVALENCE OF HPV-POSITIVE OROPHARYNGEAL CANCERS AMONG OLDER ADULTS." Cancer 124 (14): 2993-2999. doi:10.1002/cncr.31385.

56. Winerdal, Malin E., Per Marits, Max Winerdal, Mudhar Hasan, Robert Rosenblatt, Anna Tolf, Katarina Selling, Amir Sherif, and Ola Winqvist. 2011. "FOXP3 and Survival in Urinary Bladder Cancer." BJU Internationa/ 108 (10): 1672-1678. doi:10.1111/j.1464-410X.2010.10020.x.

57. Yamazaki, Sayuri, Akira Maruyama, Kohei Okada, Misako Matsumoto, Akimichi Morita, and Tsukasa Seya. 2012. "Dendritic Cells from Oral Cavity Induce Foxp3(+) Regulatory T Cells upon Antigen Stimulation." PloS One 7 (12): e51665. doi:10.1371/journal.pone.0051665.

58. Young, David, Christopher C. Xiao, Benjamin Murphy, Michael Moore, Carole Fakhry, and Terry A. Day. 2015. "Increase in Head and Neck Cancer in Younger Patients Due to Human Papillomavirus (HPV)." Oral Oncology 51 (8): 727-730. doi:10.1016/j.oraloncology.2015.03.015.

59. Zhang, Xue-Mei, Ling-Jun Song, Juan Shen, Hao Yue, Ya-Qin Han, Chen-Ling Yang, Shi-Yun Liu, et al. 2018. "Prognostic and Predictive Values of Immune Infiltrate in Patients with Head and Neck Squamous Cell Carcinoma." Human Pathology 82: 104-112. doi:10.1016/j.humpath.2018.07.012.

60. Zhou, Chen, Pengfei Diao, Yaping Wu, Zicheng Wei, Lei Jiang, Wei Zhang, Zhongwu Li, et al. 2020. "Development and Validation of a Seven-Immune-Feature-Based Prognostic Score for Oral Squamous Cell Carcinoma after Curative Resection." International Journal of Cancer 146 (4): 11521163. doi:10.1002/ijc.32571. 


\section{Supplementary Data}

Supplemental Table 1 and Figure 1 not available with this version

\section{Figures}
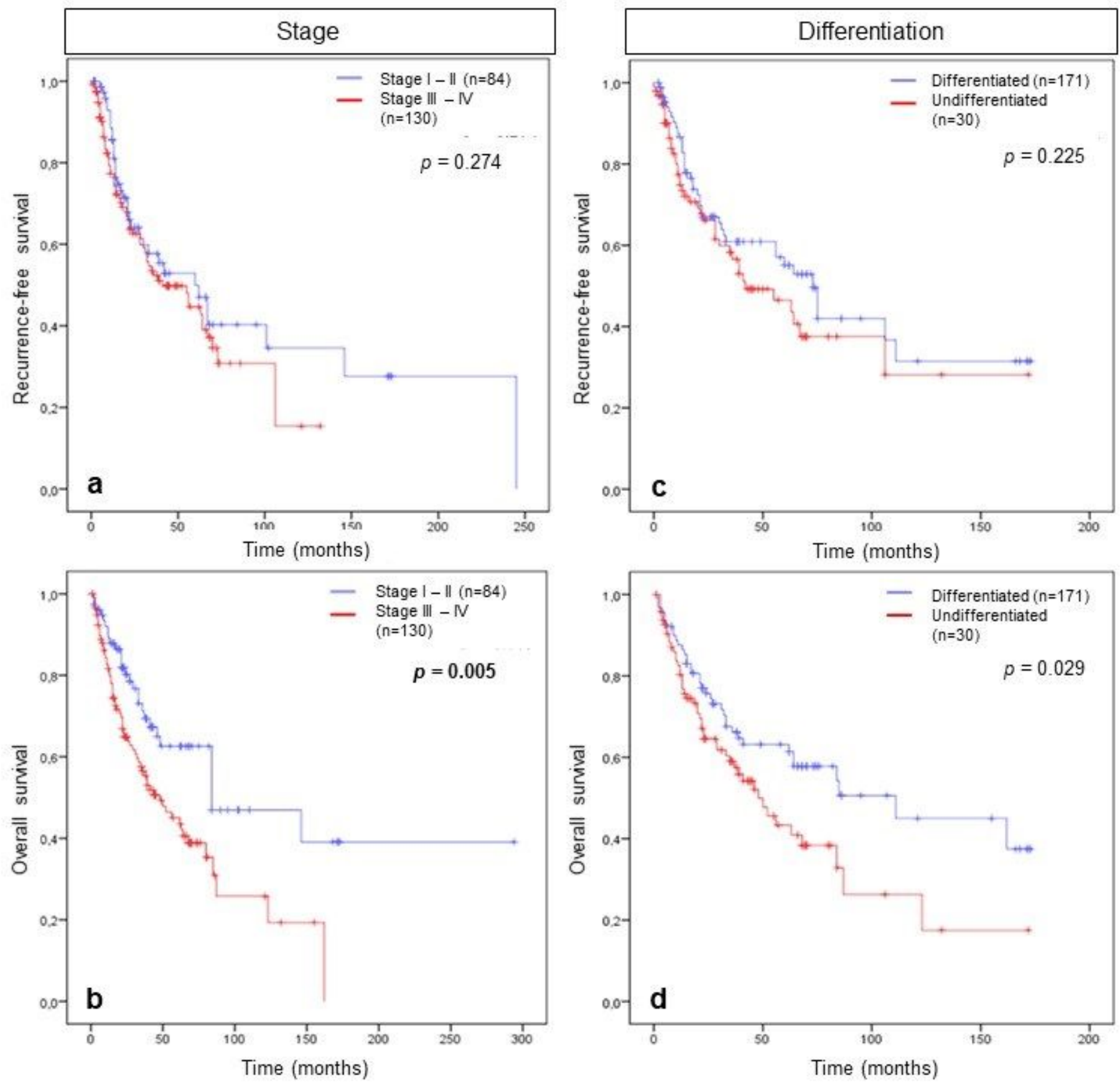

Figure 1

Association between tumour stage and differentiation and patient survival. Kaplan Meier curves comparing (a) recurrence-free survival (RFS) and (b) overall survival (OS) of tumour stage and (c) RFS 
and (d) OS of tumour differentiation.
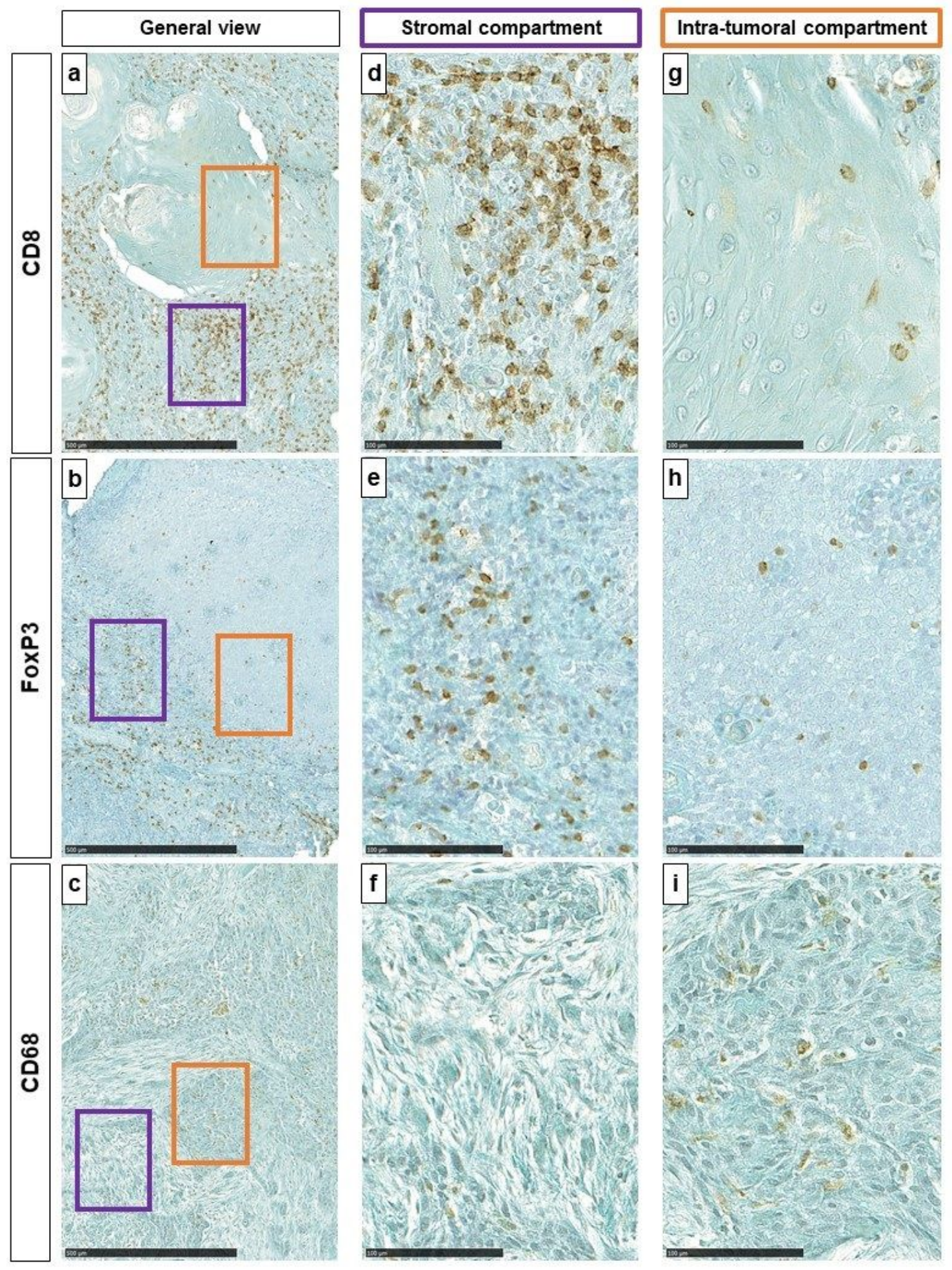

\section{Figure 2}

( $a, b$ and $c$ respectively) General view of CD8, FoxP3 and CD68 immunohistochemical staining (100x, scale $=500 \mu \mathrm{m}$ ). Purple oblong represents stromal (ST) area and orange oblong represents intra-tumoral (IT) area. (d, e, and f respectively) Representative images of CD8, FoxP3 and CD68 immuno-marker (400x, scale $=100 \mu \mathrm{m})$ in the ST and $(\mathrm{G}, \mathrm{H}$, and I respectively) CD8, FoxP3 and CD68 immuno-marker in the IT. 

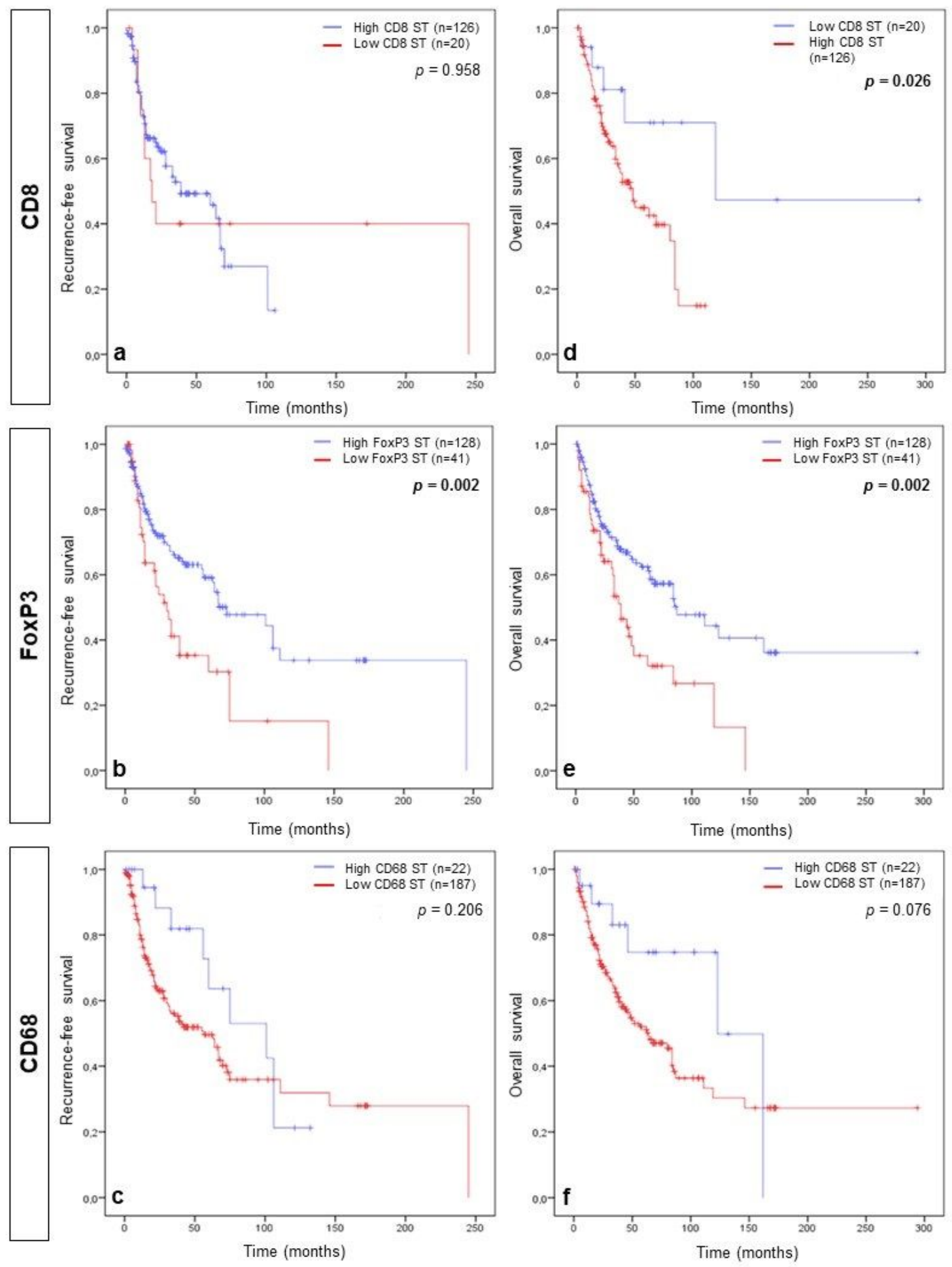

\section{Figure 3}

Association between stromal immune cells and patient survival. Kaplan Meier curves comparing $(a, b$ and c, respectively) recurrence-free survival (RFS) and CD8, FoxP3 and CD68 and (d, e and f, respectively) overall survival (OS) and CD8, FoxP3 and CD68 in the stromal compartment (ST). 

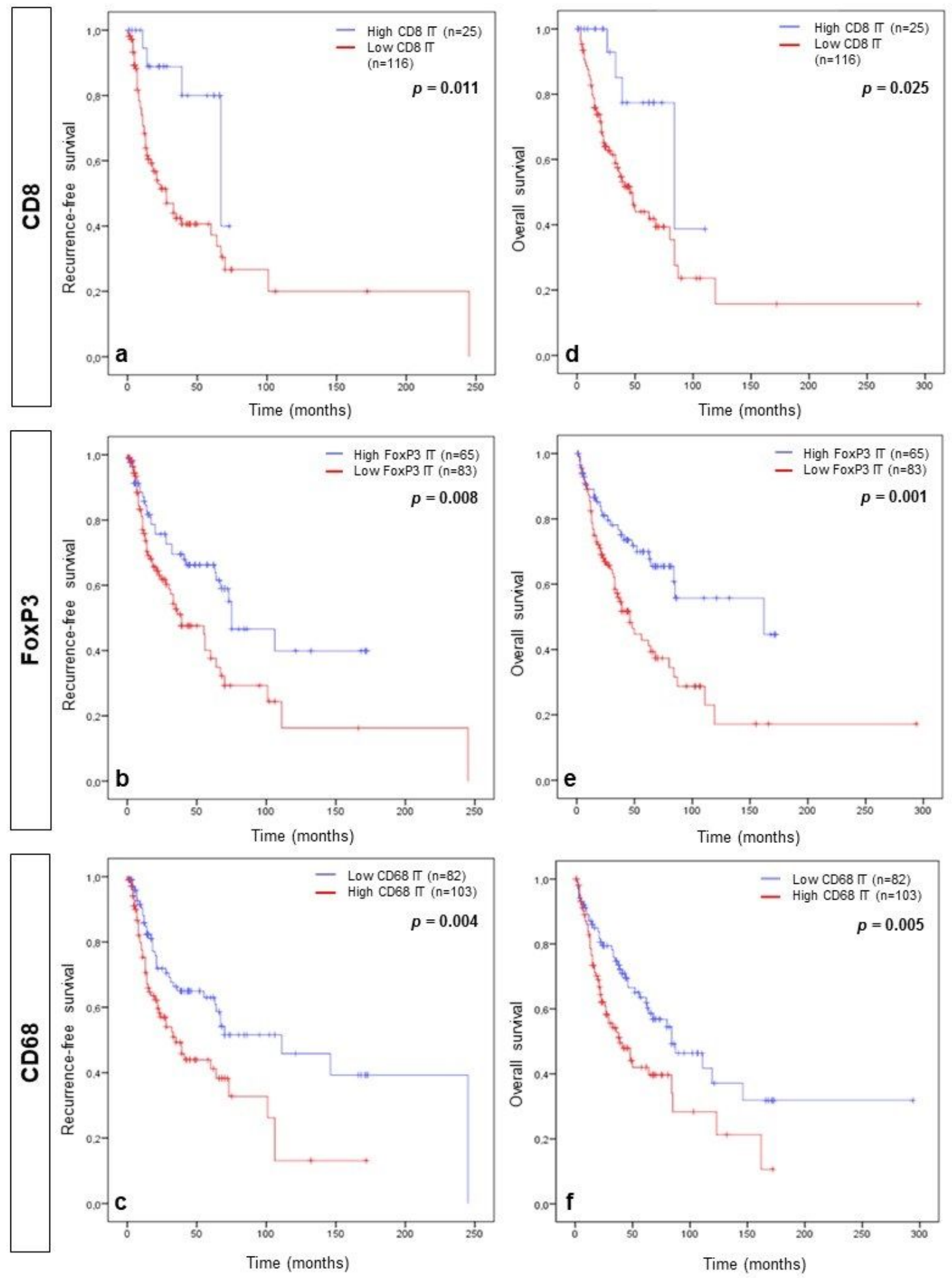

\section{Figure 4}

Association between intra-tumoral immune cells and patient survival. Kaplan Meier curves comparing (a, b and c, respectively) recurrence-free survival (RFS) and CD8, FoxP3 and CD68 and (d, e and f, respectively) overall survival (OS) and CD8, FoxP3 and CD68 in the intra-tumoral compartment (IT). 

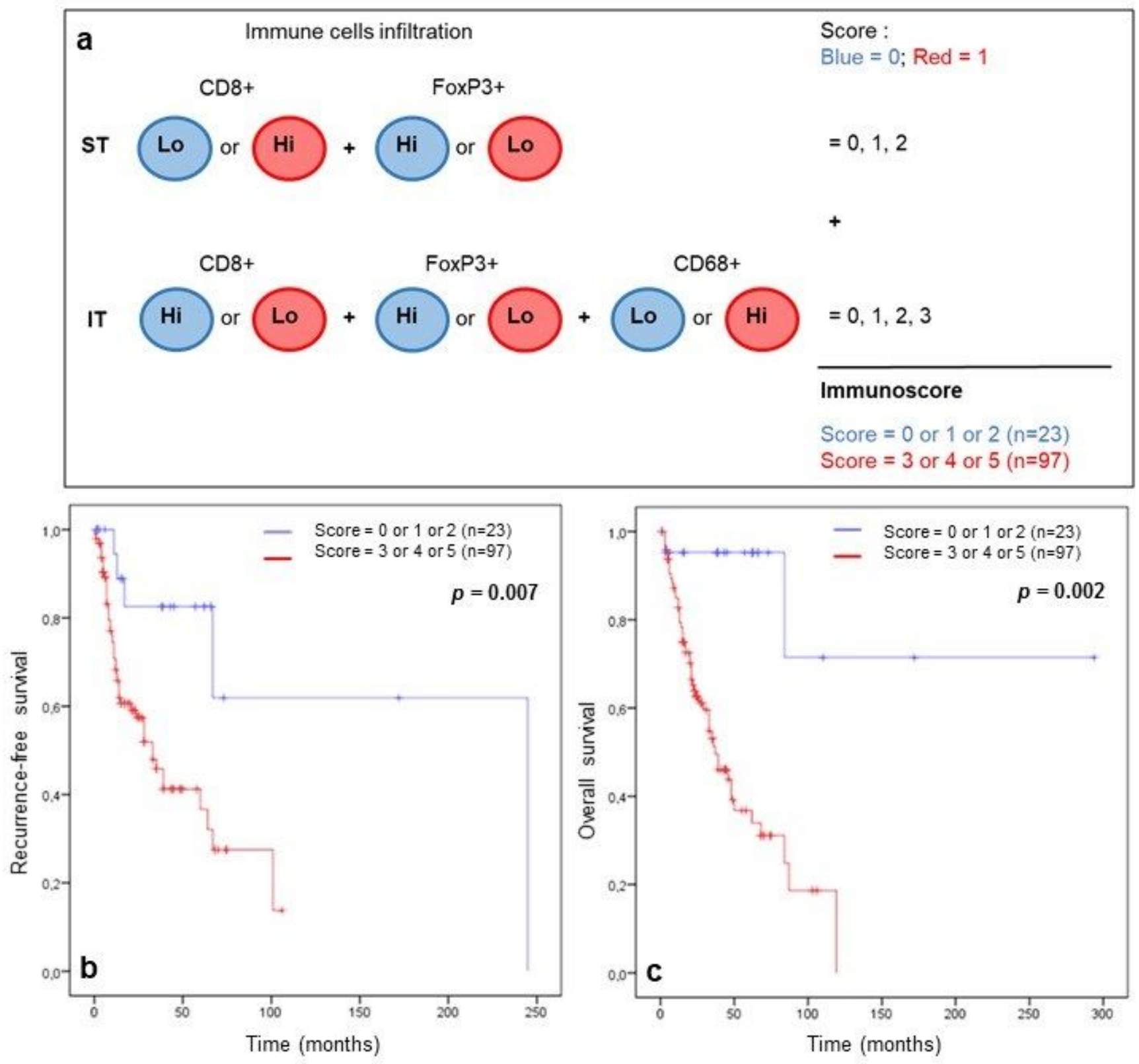

Figure 5

Immunoscore and patient survival. (a) Establishment of the immunoscore in HNSCC tissues based on the immune cells infiltration in the ST and IT compartment. (b) Kaplan Meier curves comparing recurrencefree survival (RFS) and immunoscore and (c) overall survival (OS) and immunoscore. 